PAR-LPTHE 02-04

\title{
Topological Gravity versus Supergravity on Manifolds with Special Holonomy
}

\author{
Laurent Baulieu ${ }^{a}$, Alessandro Tanzini ${ }^{a, b}$ \\ a Laboratoire de Physique Théorique et Hautes Energies, \\ Universités Pierre et Marie Curie, Paris VI \\ et Denis Diderot, Paris VII \\ ${ }^{b}$ Istituto Nazionale di Fisica Nucleare, Roma
}

\begin{abstract}
We construct a topological theory for euclidean gravity in four dimensions, by enforcing self-duality conditions on the spin connection. The corresponding topological symmetry is associated to the $S U(2) \times$ diffeomorphism $\times U(1)$ invariance. The action of this theory is that of $d=4, N=2$ supergravity, up to a twist. The topological field theory is $S U(2) \subset S O(4)$ invariant, but the full $S O(4)$ invariance is recovered after untwist. This suggest that the topological gravity is relevant for manifolds with special holonomy. The situation is comparable to that of the topological Yang-Mills theory in eight dimensions, for which the $S O(8)$ invariance is broken down to $\operatorname{Spin}(7)$, but is recovered after untwisting the topological theory.
\end{abstract}

Postal address: Laboratoire de Physique Théorique et des Hautes Energies, Unité Mixte de Recherche CNRS 7589, Université Pierre et Marie Curie, boîte postale 126.

4, place Jussieu, F-75252 PARIS Cedex 05 


\section{Introduction}

Self-duality equations play an important role in the context of topological quantum field theory (TQFT). They provide topological gauge functions that one enforces in a BRST invariant way, which determine in turn a supersymmetric action in a twisted form. In [1], there is a classification for possible self-duality equations for the curvatures of forms of degree $p$ in spaces with dimension $d \leq 16$. For Euclidean gravity in four dimensions, it has been known for a long time that the self-duality equations solving the Einstein equations involve the spin-connection itself [2]. This apparent lack of gauge independence of a physical equation is not harmful, since its solution can be related to a physical solution - the gravitational instanton- by a well-defined Lorentz transformation.

In eight dimensions, an interesting result was found, namely that the eight-dimensional topological Yang-Mills theory gives a $S O(8)$ covariant theory which is $\operatorname{Spin}(7)$ invariant, but is a twisted version of the eightdimensional supersymmetric Yang-Mills theory [3] [4] औ. By analogy, we expect that topological gravities can only be relevant for manifolds with special holonomy. Indeed, the possible gravitational self-duality equations for the spin connection can only be invariant under a subgroup of the Lorentz group (for instance $S U(2) \subset S O(4)$ in four dimensions, $\operatorname{Spin}(7) \subset S O(8)$ in eight dimensions). In the case of topological Yang-Mills theories, there are arguments according to which the full Lorentz invariance can be recovered, after untwisting the topological field theory into a supersymmetric theory. In the case of topological gravities, the situation is more involved, because the gauge group is mainly the Lorentz $\times$ diffeomorphism symmetry which must be linked to local supersymmetry instead of global supersymmetry. It is a priori not clear whether the untwisting procedure can be systematically applied in a way that transforms the topological gravity into its natural image, $N=2$ supergravity. This has been shown to be true in two dimensions [6], and Anselmi and Frè have given good arguments that the four-dimensional $N=2$ supergravity can be twisted in a topological gravity [7]. There is moreover a quite general argument that makes us confident that one can reach

\footnotetext{
${ }^{1}$ By dimensional reduction to four dimensions and a suitable gauge-fixing in the Cartan algebra allowed by topological invariance, this theory gives the abelian monopole theory of Seiberg and Witten [5], which describes the infra-red behavior of the non-abelian Super Yang-Mills theory with extended supersymmetry [3].
} 
all $N=2$ supergravities, through the construction of topological gravities coupled to TQFT's for $p$-form gauge fields. Indeed, all types of superstring theories can be obtained by suitable anomaly free untwisting of topological sigma-models, which are quite easy to derive, at least in a formal way [\&]. Then, we can rely on the fact that supergravities arise as low energy limits of superstrings in order to predict a link between supergravities and topological gravities.

The aim of this paper is to give a direct construction of the topological gravity in four dimensions, and then to show how it determines the $N=2, d=4$ supergravity. As we will see, manifolds with special holonomy play a relevant rôle both in the correct definition of the topological theory and of the twist. A nice feature of our work is that it gives a geometrical interpretation of the graviphoton in supergravity. Another interest is that it establishes a solid framework for generalizations in higher dimensions. In the concluding remarks, we will comment about a possible generalization of our topological action in eight dimensions, which should be relevant for manifolds with $\operatorname{Spin}(7)$ holonomy.

As for the determination of new invariants in four dimensions, the TQFT is well defined on manifolds with an $S U(2)$ holonomy group, as we will show in Sect.3. These manifolds admit two covariantly constant chiral spinors. The determination of observables stems from descent equations, as it is standard in the BRST formalism, and describe a cohomology which is equivariant with respect to the $S U(2) \times$ diffeomorphism symmetry. Their mean values can only depend on global properties of the chosen manifold for which one computes the action and the TQFT.

\section{The topological gravity}

Let us consider the symmetries of ordinary gravity in four dimensions. The basic symmetry is the Lorentz $\times$ diffeomorphism symmetry, whose gauge fields are the spin connection $\omega_{\mu}^{a b}$ and the vielbein $e_{\mu}^{a}$. We consider these fields as independent ones in a first order formalism, and through the paper the latin indices $a, b, \ldots$ denote flat $S O(4)$ tangent space indices, and $\mu, \nu, \ldots$ are world indices. The curvatures are

$$
R^{a b}=d \omega^{a b}+\omega^{a c} \wedge \omega^{c b}, \quad T^{a}=d e^{a}+\omega^{a c} \wedge e^{c},
$$


where $D=d+[\omega, \bullet]$ is the Lorentz covariant derivative. We define the Lie derivative as $\mathcal{L}_{\xi}=i_{\xi} d-d i_{\xi}$, where $\xi^{\mu}$ is an anticommuting vector field. We could as well write the following equation by redefining $\mathcal{L}_{\xi}$ into the operator $i_{\xi} D-D i_{\xi}$. The equations remain the same, up to a redefinition of all ghosts and of ghost of ghosts, using the similarity operation $\exp i_{\xi}$ [9]. With the covariant constraint $T^{a}=0$, one has $\omega=\omega(e)$, which determines $\omega$ up to a local Lorentz transformation. Eventually, a more refined version of this constraint will be enforced in a BRST invariant way in Section 5 .

Since the Lorentz $\times$ diffeomorphism symmetry determines a Lie group, the topological BRST symmetry can be straightforwardly constructed as:

$$
\begin{aligned}
s e_{\mu}^{a} & =\mathcal{L}_{\xi} e_{\mu}^{a}-\Omega^{a b} e_{\mu}^{b}+\Psi_{\mu}^{a} \\
s \omega_{\mu}^{a b} & =\mathcal{L}_{\xi} \omega_{\mu}^{a b}+D_{\mu} \Omega^{a b}+\tilde{\Psi}_{\mu}^{a b} \\
s \Psi_{\mu}^{a} & =\mathcal{L}_{\xi} \Psi_{\mu}^{a}-\Omega^{a b} \Psi_{\mu}^{b}-\mathcal{L}_{\Phi} e_{\mu}^{a}+\tilde{\Phi}^{a b} e_{\mu}^{b} \\
s \tilde{\Psi}_{\mu}^{a b} & =\mathcal{L}_{\xi} \tilde{\Psi}_{\mu}^{a b}-\Omega^{a c} \tilde{\Psi}^{c b}+D_{\mu} \tilde{\Phi}^{a b}-\mathcal{L}_{\Phi} \omega_{\mu}^{a b} \\
s \Phi^{a} & =\mathcal{L}_{\xi} \Phi^{a}-\Omega^{a c} \Phi^{a} \\
s \tilde{\Phi}^{a b} & =\mathcal{L}_{\xi} \tilde{\Phi}^{a b}-\Omega^{a c} \tilde{\Phi}^{c b} \\
s \xi^{\mu} & =\Phi^{\mu}+\xi^{\nu} \partial_{\nu} \xi^{\mu} \\
s \Omega^{a b} & =\mathcal{L}_{\xi} \Omega^{a b}-\Omega^{a c} \Omega^{c b}+\tilde{\Phi}^{a b} .
\end{aligned}
$$

Moreover, we have that $\Phi^{a}=e_{\mu}^{a} \Phi^{\mu}$, and thus, $s \Phi^{\mu}=\mathcal{L}_{\xi} \Phi^{\mu}$.

Here the fields $\Psi$ and $\tilde{\Psi}$ are, respectively, the topological ghosts for $e$ and $\omega$ and $\Phi$ and $\tilde{\Phi}$ are their ghosts of ghosts. We have that

$$
s^{2}=0 \quad\left(s-\mathcal{L}_{\xi}\right)^{2}=\mathcal{L}_{\Phi}
$$

on all fields. These equations are the structure equations for topological gravity. They can be cast in the following geometrical form, which generalizes that given in [10]:

$$
\begin{aligned}
& (s+d) e^{a}+\left(\omega^{a b}+\Omega^{a b}\right) e^{b}=\exp i_{\xi}\left(T^{a}+\Psi^{a}+\Phi^{a}\right) \\
& (s+d)\left(\omega^{a b}+\Omega^{a b}\right)+\left(\omega^{a c}+\Omega^{a c}\right) \wedge\left(\omega^{c b}+\Omega^{c b}\right)=\exp i_{\xi}\left(R^{a b}+\tilde{\Psi}^{a b}+\tilde{\Phi}^{a b}\right) .
\end{aligned}
$$

It allows for the geometrical interpretation of all fields. 


\section{The graviphoton}

To determine a TQFT, we have to choose a topological gauge function. The present paradigm is that the square of the gauge function determines the bosonic part of the TQFT action, up to boundary terms, as in [1]. In the case of gravity in four dimensions, there is a well known fact, which one uses for proving the positivity of the gravitational constant. It says that, up to a pure derivative, the Einstein Lagrangian can be expressed as a quadratic form in the Christoffel coefficients. In a first order formalism, this translates into the following equation, which is satisfied by the Einstein-Hilbert lagrangian:

$$
\mathcal{L}_{E H}=\frac{1}{4} \epsilon_{a b c d} R^{a b} \wedge e^{c} \wedge e^{d}=\omega^{a b^{-}} \wedge e^{b} \wedge \omega^{a c-} \wedge e^{c}+d\left(\omega^{a b^{-}} \wedge e^{a} \wedge e^{b}\right) .
$$

For any given Lorentz tensor $X^{a b}$, we define its self-dual or antiself-dual projection as $X^{a b^{ \pm}}=\frac{1}{2}\left(X^{a b} \pm \frac{1}{2} \epsilon^{a b c d} X^{c d}\right)$. Eq. (5) is analogous to the equation $|F|^{2}=\left|F^{ \pm}\right|^{2} \pm F \wedge F$, which allows one to express the Yang-Mills action as the square of the self-dual part of the curvature, plus a boundary term. However, the case of gravity is more subtle, since the decomposition in Eq.(5) is only $S U_{+}(2) \subset \operatorname{Spin}(4)$ invariant 2 . This is related to the fact that the internal symmetry group cannot be disentangled from the diffeomorphism symmetry, a situation that also complicates the current ideas about the twist of supersymmetry. Actually, in order that each term in the r.h.s. of Eq. (5) is globally well defined, one has to define the vierbein $e$ on manifolds with $S U(2)$ holonomy. Indeed, as we will see shortly, this allows one to define transition functions, such that $\omega^{a b^{-}} \wedge e^{b} \wedge \omega^{a c-} \wedge e^{c}$, and the boundary term in Eq. (5) can be separately integrated to give a well defined action, which one can insert in the path integral. We remark that the breaking of the full Lorentz symmetry into a subgroup by the self-duality equation, and by the decomposition of the action into a sum of a boundary term plus a square, is a phenomenon which also occurs in the eight-dimensional Yang-Mills theory, and in the more general cases classified in [1].

To define topological gravity, Eq.(5) suggests that we choose the following topological gauge function - the gravitational self-duality equation - for the vielbein:

$$
\omega_{\mu}^{a b^{-}}(e)=0
$$

\footnotetext{
${ }^{2}$ Our notation is $\operatorname{Spin}(4)=S U(2)_{+} \times S U(2)_{-}$, where $S U(2)_{+}$has self-dual generators $\left(\sigma^{a b}\right)_{\alpha}^{\beta}$ and $S U(2)_{-}$anti-self-dual generators $\left(\bar{\sigma}^{a b}\right)_{\dot{\alpha}}^{\dot{\beta}}$.
} 
Any given solution of Eq.(6) extremizes the Einstein action, according to Eq.(5). As explained in [2], although this equation is only $S U_{+}(2)$ invariant, it determines solutions of the $S O(4)$ invariant equation $R^{a b^{-}}(\omega(e))=0$. Manifolds which admit metrics satisfying this condition have an $S U(2)_{+}$ holonomy group (they admit two covariantly constant chiral spinors); then, the transition functions for the vierbeins $e_{\mu}^{a}$ can be chosen as elements of $S U(2)_{+}$. Correspondingly, by using the torsion-free condition $T^{a}=0$ to define the $\omega^{a b}=\omega^{a b}(e)$, one can easily show that the self-dual part $\omega^{a b+}(e)$ transforms as a connection, while the anti-self-dual part $\omega^{a b-}(e)$ is globally defined under the $S U(2)_{+}$local transformations. Thus each term on the r.h.s. of Eq.(司) is well-defined on these manifolds.

We then conclude that the gravitational TQFT that explores the fluctuations of the metrics around such self-dual solutions can be meaningful only when it is defined over a manifold with an $S U(2)$ holonomy group. This is analogous to what happens for the topological Yang-Mills theory in eight dimensions, for which the manifold must have $\operatorname{Spin}(7)$ or $S U(4)$ holonomy [司].

The topological gravity will describe the moduli space associated to Eq.(6), which can be rewritten as :

$$
B^{a b^{-}} e^{b} \wedge e^{c} \wedge \omega^{c a}=0
$$

where $B^{a b^{-}}$is a constant anti-self-dual Lorentz two-form. $B^{a b^{-}}$can be constructed in terms of the two constant chiral spinors of the manifold. Let us call $\Psi=\delta e$ an element of the tangent bundle of the moduli space of the gravitational instanton, solution of Eq.(可). By using the identity $\delta\left(e^{[a} \omega^{b] c} e^{c}\right)=d\left(e^{[a} \delta e^{b]}\right)$, one finds that $\Psi$ satisfies :

$$
B^{a b^{-}} d\left(e^{[a} \Psi^{b]}\right)=0 .
$$

Thus, in addition to the zero modes related to reparametrization invariance, we have the following zero modes for $\Psi$ :

$$
\Psi^{a} \rightarrow \Psi^{a}+M^{a b^{+}} e^{b}
$$

where $M^{a b^{+}}(x)$ is an arbitrary self-dual local parameter. The zero modes of Eq.(9) reflect the $S U(2)_{+}$invariance of Eq.(6), which determines the vierbein $e_{\mu}^{a}$ only up to a local $S U(2)_{+}$transformation. Actually, Eq.(96) has already 
been captured in the BRST symmetry equation for $\Psi$ in Eq.(2), and it is the reminder of this BRST equation after the breaking of the Lorentz symmetry down to $S U_{+}(2)$ by Eq.(17), with $M^{a b^{+}}=\tilde{\Phi}^{a b^{+}}$.

One subtlety of topological gravity is the way we will gauge fix the invariance in the ghost action corresponding to Eq.(9). Since $M^{a b^{+}}$is self-dual, it corresponds to three degrees of freedom and one must introduce Lagrange multipliers that count for three independent gauge-fixings. However, we can associate to $M^{a b+}$ a one-form defined modulo gauge transformation, which also counts for $3=4-1$ degrees of freedom. Thus, we will introduce an antighost $A$ that is an abelian one-form, whose BRST variation is a fermionic abelian one-form $\bar{\Psi}$. Thus, the very basic invariance in Eq.(9) strongly suggest to introduce a $U(1)$ graviphoton field $A_{\mu}$ in topological gravity, with the interpretation that $A_{\mu}$ has ghost number -2 for consistency. The action must only depend on $A$ through $F=d A$, so that we expect that all ghosts associated to the $S U(2)_{+} \times$diffeomorphism invariance carry no charge under this additional $U(1)$ symmetry.

From a geometrical point of view, for manifolds with a metric satisfying Eq.(6), the existence of the zero modes (9) is related to the existence of a certain number of self-dual harmonic two-forms, equal to the Hirzebruch signature $\tau$ of the manifold $\mathcal{M}$. For the simplest example, the EguchiHanson gravitational instanton, one has only one such self-dual two-form. In general, one has that, locally, $M^{a b^{+}} e^{a} \wedge e^{b}=\sum_{i=1}^{\tau} c_{i} h^{i}$, where $h^{i}$ is a basis of the cohomology group $H^{2+}(\mathcal{M}, \mathbb{R})$. Correspondingly, one has to introduce a series of Maxwell fields $A^{i}, i=1, \ldots, \tau$, with ghost number -2 . This can be done by adding to the topological gravity action a sum of topological Maxwell actions, which are constructed in a standard way from the selfduality conditions on the curvatures $F^{i}=d A^{i}$ of the additional $U(1)$ gauge fields.

The above considerations lead us to complete the BRST equations by introducing the $U(1)$ graviphoton $A$. To do so, we must also introduce an abelian ghost $c$, a topological ghost $\bar{\Psi}$ and a ghost of ghost $\Phi$, so that:

$$
\begin{aligned}
s A_{\mu} & =\mathcal{L}_{\xi} A_{\mu}+\partial_{\mu} c+\bar{\Psi}_{\mu} \\
s \bar{\Psi}_{\mu} & =\mathcal{L}_{\xi} \bar{\Psi}_{\mu}-\partial_{\mu} \Phi-\mathcal{L}_{\Phi} A_{\mu} \\
s \Phi & =\mathcal{L}_{\xi} \Phi-\mathcal{L}_{\Phi} c \\
s c & =\mathcal{L}_{\xi} c+\Phi
\end{aligned}
$$


and:

$$
(s+d)(A+c)=\exp i_{\xi}(F+\bar{\Psi}+\Phi)
$$

The ghost numbers of the fields $A, \bar{\Psi}, \Phi$, and $c$ are $-2,-1,0$ and -1 , respectively. Thus, the BRST symmetry that we consider corresponds to the topological symmetry for the Lie algebra of the $S U(2)_{+} \times$diffeomorphism $\times U(1)$ group. Since the diffeomorphisms cannot be represented by finite matrices, we expect that the untwist of the topological symmetry should determine $N=2$ local supersymmetry, instead of global supersymmetry.

If one sets equal to zero the ghosts $\xi^{\mu}, \Omega^{a b}$ and $c$ in all BRST equations, one gets a BRST operator that is nilpotent, modulo a diffeomorphism with parameter $\Phi^{\mu}=e_{a}^{\mu} \Phi^{a}$, a Lorentz transformation with parameter $\tilde{\Phi}^{a b}$ and a $U(1)$ transformation with parameter $\Phi$. The cohomology of this operator define the observables of the topological theory.

\section{The antighost sector}

In order to perform the topological gauge-fixing that realizes a TQFT around the solution of Eq.(6), we need to complete the fields that have been introduced above, by introducing suitable antighosts and lagrangian multipliers. Some of the latter will actually be propagating fields, a situation that is a current one in supergravity. Moreover, only the $S U(2)_{+}$sector of the Lorentz group, which is left invariant by the gauge-fixing condition Eq.(6), has to be retained. The corresponding ghosts are given by the self-dual parts of the fields $\Omega^{a b}, \tilde{\Phi}^{a b}$ considered in Sect. 2 .

To display the fields, we adopt the presentation of [10]. In the following diagrams, the ghost number is the same in any given column, and decreases by one unit when one goes from one column to the next one on the right. We have made the world indices explicit. Notice that $e$ and $\omega$ have ghost number zero, while $A$ has ghost number -2; this implies in particular that the BRST doublet $\left(\chi^{a b^{+}}, \beta^{a b^{+}}\right)$has positive ghost numbers equal to $(1,2)$ respectively. The following triangular presentation of all fields can be justified if one introduces an additional grading that accounts for the antighost number, which 
allows one to generalize the geometrical equations (41) and (10). We have

$$
\begin{aligned}
& \begin{array}{ccccc} 
& & e_{\mu}^{a} & & \\
\Phi^{a} & \Psi_{\mu}^{a} & & \bar{\Psi}_{\mu}^{a b^{-}} & \\
& \eta^{a b^{-}} & L^{a b^{-}}, b_{\mu}^{a b^{-}} & & \bar{\Phi}^{a} \\
& & \bar{\eta}^{a} &
\end{array} \\
& \begin{array}{ccccc} 
& & \omega_{\mu}^{a b} & & \\
& \tilde{\Psi}_{\mu}^{a b} & & \overline{\tilde{\Psi}}_{\mu}^{a b} & \\
\tilde{\Phi}^{a b^{+}} & & \tilde{L}^{a b^{-}}, \tilde{b}_{\mu}^{a b} & & \overline{\tilde{\Phi}}^{a b^{+}} \\
& \tilde{\eta}^{a b^{-}} & & \bar{\eta}^{a b^{+}} &
\end{array}
\end{aligned}
$$

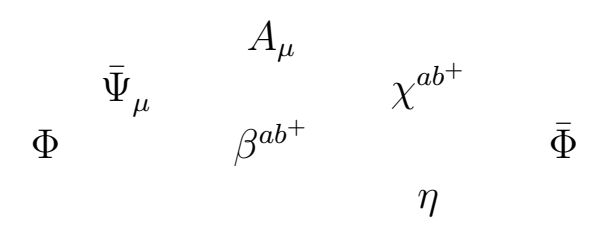

$$
\begin{aligned}
& \xi^{\mu}{ }_{b^{\mu}} \bar{\xi}^{\mu} \quad \Omega^{a b^{+}}{ }_{b^{a b^{+}}} \bar{\Omega}^{a b^{+}} \quad c \quad{ }{ }^{\bar{c}}
\end{aligned}
$$

Clearly, the antighost $\bar{\Psi}_{\mu}^{a b^{-}}$and its Lagrange multiplier $b_{\mu}^{a b^{-}}$are introduced to implement the gauge condition on $\omega_{\mu}^{a b^{-}}$as given by Eq.(6), while $\overline{\tilde{\Psi}}_{\mu}^{a b}$ and its Lagrange multiplier $\tilde{b}_{\mu}^{a b}$ will be used to impose, in BRST invariant way, the relevant gauge condition on the torsion $T_{\mu \nu}^{a}(e, \omega)$, which allows one to express the spin connection as a function of $e$, and possibly of other fields. The rest of the fields are needed because we are building a TQFT that is equivariant with respect to the $S U(2)_{+} \times$diffeomorphism $\times U(1)$ symmetry. This means that the gauge functions on the fields have an internal gauge symmetry, which must fixed. To do so, one needs suitable ghosts for ghosts, antighosts for antighosts and their Lagrange multipliers, which allow one to remove the degeneracies of all ghost propagators, such as the one displayed in Eq.(9).

All equations for the antighosts appearing in our field spectrum are of the type

$$
\hat{s} \bar{g}=\lambda \quad \hat{s} \lambda=\mathcal{L}_{\Phi} \bar{g}+\delta_{\tilde{\Phi}} \bar{g}
$$


with $s X=\hat{s} X+\mathcal{L}_{\xi} X+\delta_{\Omega^{+}} X$. Notice that $\hat{s}^{2}=\mathcal{L}_{\Phi}+\delta_{\tilde{\Phi}^{+}}$, where $\delta_{\tilde{\Phi}^{+}}$is a Lorentz transformation with parameter $\tilde{\Phi}^{a b^{+}}$, and that none of the antighosts transform under the $U(1)$ symmetry defined in Eq.(10). We have :

$$
\begin{gathered}
\hat{s} \bar{\Psi}_{\mu}^{a b^{-}}=b_{\mu}^{a b^{-}} \quad \hat{s} L^{a b^{-}}=\eta^{a b^{-}} \quad \hat{s} \bar{\Phi}^{a}=\bar{\eta}^{a} \\
\hat{s} \overline{\tilde{\Psi}}_{\mu}^{a b}=\tilde{b}_{\mu}^{a b} \quad \hat{s} \tilde{L}^{a b^{-}}=\tilde{\eta}^{a b^{-}} \quad \hat{s} \overline{\tilde{\Phi}}^{a b^{+}}=\overline{\tilde{\eta}}^{a b^{+}} \\
\hat{s} \chi^{a b^{+}}=\beta^{a b^{+}} \quad \hat{s} \bar{\Phi}=\eta \\
\hat{s} \bar{\xi}^{\mu}=b^{\mu} .
\end{gathered}
$$

It is convenient to do field redefinitions that express the BRST transformation for the antighost $\bar{\Psi}_{\mu}^{a b^{-}}$as follows:

$$
\begin{aligned}
\hat{s} \bar{\Psi}_{\mu}^{a b^{-}} & =b_{\mu}^{a b^{-}}+\partial_{\mu} L^{a b^{-}}, \\
\hat{s} b_{\mu}^{a b^{-}} & =\left(\mathcal{L}_{\Phi}+\delta_{\tilde{\Phi}^{+}}\right) \bar{\Psi}_{\mu}^{a b^{-}}+\partial_{\mu} \eta^{a b^{-}}-\left[\tilde{\Psi}_{\mu}, L\right]^{a b^{-}} .
\end{aligned}
$$

In this way one can take care in a more transparent way of the degeneracy which occurs in the term $\bar{\Psi}^{a b^{-}} \wedge s\left(e^{[a} \omega^{b] c} e^{c}\right)=\bar{\Psi}^{a b^{-}} \wedge d\left(e^{[a} \wedge \Psi^{b]}\right)$ of the action (25). The corresponding zero-modes for $\bar{\Psi}_{\mu}^{a b^{-}}$are explicit in Eq.(20).

\section{The topological gravity action}

In order to determine a TQFT for topological gravity, which uses the above fields, we need a little bit of thinking. The expression Eq.(可) of the Einstein action and the the self-duality equation (6) are the signal for the existence of a TQFT for gravity. It is quite clear that the definition of a TQFT that concentrates in a BRST invariant way the path integral around the solution of Eq.(6) breaks the $S O(4)$ invariance down to $S U_{+}(2)$. We must therefore consider a quantum field theory in which the path integral measure only involves vierbeins that determine a globally well defined topological Lagrangian. As discussed in Sect.3, this restricts the choice to manifolds with $S U(2)$ holonomy. 
To recover the full Lorentz symmetry after an untwisting procedure, the existence of the topological ghost $\bar{\Psi}$ of the $U(1)$ gauge field $A$ is essential. It allows a complete determination of spinor fields. Indeed, the fields

$$
\left(\Psi_{\mu}^{a}, \bar{\Psi}_{\mu}^{a b^{-}}, \bar{\Psi}_{\mu}\right)
$$

can be untwisted to the two gravitini $\left(\lambda_{\mu}^{\dot{\alpha} i}, \lambda_{\mu \alpha}^{i}\right)$, where $i=1,2$ labels the automorphism group $S U(2)_{R}$ of $N=2$ supergravity. As shown explicitely in the next section, this follows from the fact that, two pairs of dotted and undotted spinors can be twisted into a vector, a selfdual or antiselfdual twoform and a scalar, thanks to the two different possibilities of extracting an $S O(4)$ symmetry from the $S U_{+}(2) \times S U_{-}(2) \times S U(2)_{R}$ symmetry group [11]. Thus, we need the Lorentz scalar ghost $\bar{\Psi}_{\mu}$, and we understand that $A_{\mu}$ must have ghost number -2 , in order that $\bar{\Psi}_{\mu}$ has ghost number -1 , as $\bar{\Psi}_{\mu}^{a{ }^{-}}$. This completes the analysis done in the previous section for the introduction of A.

In supergravity, the standard gauge condition for the local supersymmetry on the spin $3 / 2$ gravitini $\lambda_{\mu}^{i}$ is

$$
\gamma^{\nu} D_{\nu} \gamma^{\mu} \lambda_{\mu}^{i}=0
$$

If this condition is imposed in a way that respects the ordinary BRST symmetry of supergravity, it yields a propagation of the Lagrange multipliers, as first observed by Nielsen and Kallosh [12]. This leaves little room, but for the interpretation of the fields

$$
\left(\Phi^{a}, L^{a b^{-}}+\tilde{L}^{a b^{-}}, \Phi\right),\left(\bar{\Phi}^{a}, L^{a b^{-}}-\tilde{L}^{a b^{-}}, \bar{\Phi}\right)
$$

as the twist of pairs of commuting Majorana spinors that will be identified, eventually, as the ghosts and antighosts of the local supersymmetry of $N=2$ supergravity. Then,

$$
\left(\bar{\eta}^{a}, \eta^{a b^{-}}, \eta\right)
$$

can be identified as the twist of anticommuting Majorana spinors, which are the propagating Lagrange multipliers for the gauge-fixing on the gravitini, as we will see in more detail in the next section. The twisted version of the gauge condition (22) naturally arises as the relevant gauge fixing for the topological ghosts that occur in topological gravity. 
This parallel is just enough to tell us how to choose the gauge functions for having a theory which, (i), concentrates the path integral around the gravitational instanton, and (ii), has a BRST symmetry corresponding to a twisted supersymmetry. Since the Lie algebra we start from contains the reparametrization symmetry, which cannot be represented by finite matrices, we expect a link between this topological BRST symmetry and local supersymmetry, rather than global supersymmetry.

We face the problem of determining an action that contains the EinsteinHilbert term, expressed under the form displayed in Eq.(5), plus a term that depends on the sixteen fermionic degrees of freedom contained in Eq.(21). This action is :

$$
\begin{aligned}
I_{T Q F T}= & \int s\left[\bar{\Psi}^{a c^{-}} \wedge e^{c} \wedge e^{b} \wedge\left(\omega^{b a^{-}}+b^{b a^{-}}\right)\right] \\
& +s\left[\chi^{+}\left(\beta^{+}+2\left(F+\bar{\Psi}^{a b^{-}} \wedge \bar{\Psi}^{a b^{-}}+\bar{\Psi} \wedge \bar{\Psi}\right)\right)+2 F^{-} \wedge \Psi^{a} \wedge e^{a}\right]+ \\
& +s\left[\overline{\tilde{\Psi}}^{a b} \wedge e^{b} \wedge^{*}\left(T^{a}(\omega, e)+\bar{\Psi}^{a b^{-}} \wedge \Psi^{b}+\bar{\Psi} \wedge \Psi^{a}\right)\right]
\end{aligned}
$$

where $\chi^{+}=\chi^{a b^{+}} e^{a} \wedge e^{b}$ and $\beta^{+}=\beta^{a b^{+}} e^{a} \wedge e^{b}$. One must expand the $s$-exact term, using the above definition of $s$, to get the full expression of $I_{T Q F T}$. Notice that the dependence on the Lagrange multiplier $\beta^{+}$, which has ghost number two, arising from the second line of Eq.(25) breaks the $U(1)$ ghost number symmetry of this action down to a $\mathbb{Z}_{2}$ symmetry. This means that the ghost number is conserved only modulo two. This is an unavoidable feature if we want the graviphoton field $A$ to propagate with a Maxwell term $F \wedge^{*} F$, since, according to our previous definitions, this term has ghost number -4 . From the twisted supergravity point of view, this can be understood as a consequence of the fact that the $U(1)_{R}$ group, which is identified with the ghost number symmetry after the twist, is only a symmetry of the equation of motions of $N=2$ supergravity, and not of the whole action [7]. However, this problem can be avoided in the topological theory, which can be alternatively defined without the term in $\left(\beta^{+}\right)^{2}$ responsible of the $U(1)$ symmetry breaking. In this case, the $A$ field does not propagate, and the topological action (25) simply localizes this field to its classical solutions.

The last term in the Eq.(25) gives a constraint that determines in a Lorentz covariant way the spin connection $\omega$ as a function of $e$ and of other fields in Eq. 21). It also allows one to eliminate the Lorentz topological ghosts $\tilde{\Psi}$ and $\tilde{\Psi}$ in function of the other ghosts by algebraic equations of 
motion.

In order to fix the gauge for the topological ghosts, we consider the following $s$-exact term :

$$
I_{\text {ghosts }}=\int d^{4} x s\left[\sqrt{g}\left(\bar{\Phi}^{a} D_{\mu} \Psi_{\mu}^{a}+\tilde{L}^{a b^{-}} D_{\mu} \bar{\Psi}_{\mu}^{a b^{-}}+\bar{\Phi} \partial_{\mu} \bar{\Psi}_{\mu}\right)\right]
$$

This term, after untwisting, provides the propagation of the fields in Eqs.(23) and (24), together with a gauge-fixing for the longitudinal parts of all topological ghosts in Eq.(21).

In order to establish the comparison with the twisted supergravity action, we need an identification of $\chi^{a b^{+}}$with $\Omega^{a b^{+}}$, which is obtained by the additional term:

$$
I_{\chi / \Omega}=\int d^{4} x s\left[\sqrt{g}\left(\overline{\tilde{\Phi}}^{a b^{+}}\left(\Omega^{a b^{+}}-\chi^{a b^{+}}\right)\right)\right] .
$$

In the next section we will see in fact that the field $\chi^{a b^{+}}$does not appear in the twisted supergravity multiplet. The term (27) also gauge-fixes the symmetry in Eq.(9), by providing an equation for the ghost-for-ghost $\tilde{\Phi}^{a b^{+}}$. Notice that the terms in $\Omega^{a b^{+}}$appearing in Eq.(25) after the identification (27) can be easily reabsorbed thanks to the $S U(2)_{+}$equivariance of the action's. In the next section we will show that the resulting topological action corresponds to the twisted version of $N=2$ pure supergravity.

As for the gauge fixing of the local Lorentz symmetry, we proceed in two steps. We know that, if the Riemann curvature is self-dual, i.e. $R^{a b^{-}}=0$,

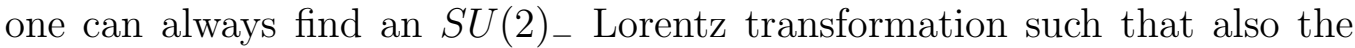
corresponding spin connection is self-dual, $\omega^{a b^{-}}=0$ [2]. The first line of Eq.(25) exactly implements this condition in an $S U(2)_{+}$equivariant way. Eventually, the left-over $S U(2)_{+}$invariance can be fixed by the term:

$$
I_{\text {Lorentz }}=\int d^{4} x s\left[\sqrt{g}\left(\bar{\Omega}^{a b^{+}}\left(e_{\mu}^{a} \delta_{b}^{\mu}-e_{a}^{\mu} \delta_{\mu}^{b}\right)\right)\right] .
$$

The action

$$
I=I_{T Q F T}+I_{\text {ghosts }}+I_{\chi / \Omega}+I_{\text {Lorentz }}
$$

is thus our candidate for describing topological gravity. It is still $U(1)$ and reparametrization invariant. The gauge fixing of these last symmetries can be

\footnotetext{
${ }^{3}$ They can actually be reabsorbed by the field redefinition $\Psi^{a \prime}=\Psi^{a}+\frac{1}{2} \Omega^{a b^{+}} e^{b}$, which does not induce additional terms in the action due to its $S U(2)_{+}$equivariance.
} 
completed by the obvious term $s\left(\bar{c} \partial_{\mu} A_{\mu}+\bar{\xi}^{\nu} \partial_{\mu} g_{\mu \nu}\right)$. A more refined analysis of this gauge fixing term could be done, by introducing the extended formalism with another BRST operation, which would control the reparametrization and $U(1)$ invariances, in a way that is analogous to that used for other types of topological gauge theories in [13].

As for the observables, the situation is as follows. We have constructed a Lagrangian that is globally well-defined over any given four-dimensional manifold $\mathcal{M}$ with $S U(2)$ holonomy. It can be considered as the gaugefixing of topological invariants, which can be combinations of $\operatorname{Tr}\left(R^{a b} \wedge R^{a b}\right)$, $\operatorname{Tr}\left(\epsilon_{a b c d} R^{a b} \wedge R^{c d}\right)$ and $F \wedge F$. The operation $s$ describes a topological symmetry that is equivariant with respect to $S U(2)+$ and reparametrization symmetry. Eq.(41) indicates that possible observables are the field polynomials obtained by doing the substitutions $T \rightarrow T+\Psi+\Phi, R \rightarrow R+\tilde{\Psi}+\tilde{\Phi}$ and $F \rightarrow F+\bar{\Psi}+\Phi$, in all invariant polynomials in $R, T$ and $F$ that one can construct, as a generalization of [3]. The claim is that the expectation values of these observables, computed from the action in Eq.(29), only depend on the differential structure of $\mathcal{M}$. The amazing feature is the relationship of the whole action to Poincaré supergravity.

\section{The relationship to the supergravity action}

We will now see that the topological gravity theory discussed in the previous section corresponds to the twisted version of four dimensional $N=2$ pure supergravity. The twist of this theory has been already considered in [7]. What we want to underline here is that special holonomy manifolds are required in order to have a well-defined twist operation. We have seen in the previous section that the topological gravity lagrangian is globally well-defined on manifolds with $S U(2)$ holonomy. The corresponding BRST algebra is associated with the $S U(2)_{+} \times$diffeomorphism $\times U(1)$ symmetry, while the re-

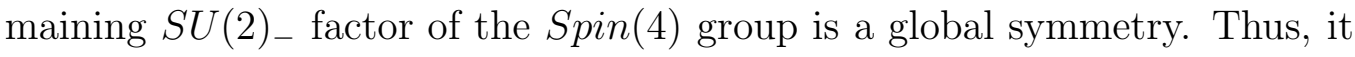
is possible to define the twist on such manifolds, as one does in the standard global supersymmetry case, by redefining a new factor $S U(2)_{-}^{\prime}$, such that:

$$
S U(2)_{-}^{\prime} \equiv \operatorname{diag}\left(S U(2)_{-} \oplus S U(2)_{R}\right)
$$

Correspondingly, the fields of the topological gravity, given by a vector, an

anti-self-dual two-form and a scalar denoted as $\left(X^{a}, \bar{X}^{a b^{-}}, \bar{X}\right)$, determine two 
dotted and undotted spinors according to :

$$
\begin{aligned}
X^{\alpha \dot{\beta}} & =X^{a}\left(\sigma^{a}\right)^{\alpha \dot{\beta}} \\
X_{\dot{\beta}}^{\dot{\alpha}} & =\bar{X}^{a b}\left(\bar{\sigma}^{a b}\right)_{\dot{\beta}}^{\dot{\alpha}}+\bar{X} \delta_{\dot{\beta}}^{\dot{\alpha}}
\end{aligned}
$$

One assembles $X^{\alpha \dot{\beta}}$ and $X_{\dot{\beta}}^{\dot{\alpha}}$ as a pair of two Majorana spinors $\lambda^{i}$, by identifying the index $\dot{\alpha}$ in Eq. (31) as an internal $S U(2)_{R}$ index $i$. The dictionary between the fields of topological gravity and of supergravity is thus:

$$
\begin{aligned}
\left(\Psi_{\mu}^{a}, \bar{\Psi}_{\mu}^{a b^{-}}, \bar{\Psi}_{\mu}\right) & \rightarrow \lambda_{\mu}^{i} \\
\left(\bar{\eta}^{a}, \eta^{a b^{-}}, \eta\right) & \rightarrow d^{i} \\
\left(\Phi^{a}, L^{a b^{-}}+\tilde{L}^{a b^{-}}, \Phi\right) & \rightarrow \kappa^{i} \\
\left(\bar{\Phi}^{a}, L^{a b^{-}}-\tilde{L}^{a b^{-}}, \bar{\Phi}\right) & \rightarrow \bar{\kappa}^{i}
\end{aligned}
$$

$\lambda_{\mu}^{i}$ stand for two spin $3 / 2$ gravitini, $\kappa^{i}$ and $\bar{\kappa}^{i}$ stand for two pairs of commuting Majorana spinors that are the spin $1 / 2$ ghosts and antighosts for $N=2$ local supersymmetry, and $d^{i}$ are the corresponding spin $1 / 2$ anticommuting Lagrange multipliers.

If we restrict to terms that are not more than quadratic in the $\Psi$ and $\bar{\Psi}$ topological ghosts, it is easy to get that, after computing the $s$-exact terms in Eq.(25), and eliminating the fields with algebraic equations of motion,

$$
\begin{aligned}
I_{T Q F T} \cong & \int \omega^{a b^{-}} \wedge e^{b} \wedge \omega^{a c^{-}} \wedge e^{c}+ \\
& -\left(d \Psi^{a}+\omega^{b a^{+}} \Psi^{b}\right) \wedge \bar{\Psi} \wedge e^{a}+ \\
& -\left(d \Psi^{a}+\omega^{b a^{+}} \Psi^{b}\right) \wedge \bar{\Psi}^{a c^{-}} \wedge e^{c}+ \\
& -F^{+} \wedge F^{+}-2 F^{+} \wedge\left(\bar{\Psi}^{a b^{-}} \wedge \bar{\Psi}^{a b^{-}}+\bar{\Psi} \wedge \bar{\Psi}\right)+ \\
& +2 F^{-} \wedge \Psi^{a} \wedge \Psi^{a} .
\end{aligned}
$$

Notice that in this action the covariant derivatives with respect to the $S U(2)_{+}$ factor of the Lorentz group appear, as expected in a construction that is $S U(2)_{+}$equivariant. Up to the boundary term $d\left(\omega^{a b^{-}} \wedge e^{a} \wedge e^{b}\right)$ coming from the decomposition (5), the action in Eq.(33) yields, after untwisting, the $N=2$ supergravity action, which reads :

$$
\begin{aligned}
I_{\text {SUGRA }} \cong & \int \frac{1}{4} \epsilon_{a b c d} R^{a b} \wedge e^{c} \wedge e^{d}+D \bar{\lambda}^{i} \wedge \gamma_{5} \gamma^{a} \lambda^{i} \wedge e^{a}-F^{+} \wedge F^{+}+ \\
& -\frac{1}{2} F \wedge \bar{\lambda}^{i} \wedge \gamma_{5} \lambda^{j} \epsilon_{i j}-\frac{1}{4} \epsilon_{a b c d} F^{a b} e^{c} \wedge e^{d} \bar{\lambda}^{i} \wedge \lambda^{j} \epsilon_{i j}
\end{aligned}
$$


up to quartic terms in the gravitini. As for these terms, they should be taken into account by a suitable redefinition of the connection $\omega^{a b}$. We recall in fact that in supergravity there are extra contributions to the torsion, such that the defining equation for the connection is actually

$$
\begin{aligned}
& d e^{a}+\omega^{a c}(e, \Psi, \bar{\Psi}) \wedge e^{c}+\frac{1}{2} \bar{\lambda}^{i} \gamma^{a} \wedge \lambda^{i}= \\
& =T^{a}(\omega, e)+\bar{\Psi}^{a b^{-}} \wedge \Psi^{b}+\bar{\Psi} \wedge \Psi^{a}=0 .
\end{aligned}
$$

Thus, the solution of Eq. (33) is a function $\omega^{a c}(e, \Psi, \bar{\Psi})$, which contributes to the presence in the action of quartic terms in the fermionic fields. Such quartic terms come from the Einstein action, as well as from the second line in the topological action Eq.(25).

This comparison allows us to truly identify the topological ghosts of $e$ and $A$ as fields that can be twisted into two gravitini. Moreover the $U(1)$ gauge field is the graviphoton field. As already remarked in the previous section, a subtle feature is that $A$ has ghost number equal to minus two, which implies that the $U(1)$ ghost number symmetry is broken to $\mathbb{Z}_{2}$.

As for the rest of the action, the terms that depend on $L$ in Eq.(25) and the Lagrangian Eq.(26) provide an action, which is, after using the correspondence Eq.(32) :

$$
\int d^{4} x \sqrt{g}\left(d^{i} \gamma^{\nu} D_{\nu} \gamma^{\mu} \lambda_{\mu}^{i}+\bar{\kappa}^{i} \gamma^{\nu} D_{\nu} \gamma^{\mu} D_{\mu} \kappa^{i}\right)
$$

This is precisely what is needed to fix local supersymmetry in supergravity, and completely identifies the ghost of ghosts, antighosts of antighosts and their Lagrange multipliers in Eq.(32) as the twisted ghosts, antighosts and Nielsen-Kallosh ghosts of supergravity.

\section{$7 \quad$ Remarks and conclusions}

It is striking that a straightforward construction of topological gravity for manifolds with $S U(2)$ holonomy can be obtained as the BRST invariant gauge-fixing of the standard invariants, $R^{a b} \wedge R^{a b}$ or $\epsilon_{a b c d} R^{a b} \wedge R^{c d}$ and $F \wedge F$. We can define observables by considering the cocycles that arise from these invariants, owing to the geometrical equations (4) and (10). This possibility was already proposed by Witten [14] in the context of Weyl gravity, and by Anselmi and Frè [7]. 
As for the utility of topological gravity, it seems that it holds only for manifolds of a certain type, namely for those that admit gravitational instantons, as hyper-Kähler manifolds. As we have seen in Section 3, the fields of the topological gravity multiplet have a nice geometrical interpretation in terms of linear deformations around the gravitational instanton 1 , which seems quite analogous to that of the fields of the topological Yang-Mills theory in four dimensions for the gauge instantons [11, 16]. The manifolds involved in our analysis admit self-dual abelian connections, which can be naturally included in our topological model. We underline that the presence of such $U(1)$ connections for these manifolds, also called "abelian instantons", makes it difficult to compute for these manifolds the Seiberg-Witten topological invariants associated to the twist of theories with $N=2$ global supersymmetry [5]. The topological gravity action, which we have discussed, could be useful to get information on these cases.

It is however unclear to us whether one can give to the four-dimensional boundary term $d\left(\omega^{a b^{-}} \wedge e^{a} \wedge e^{b}\right)$ any interpretation as a topological term. This is maybe possible on certain manifolds, e.g., hyper-Kähler ones. Were it the case, it would provide cocycles from descent equations, which could be used tentatively to compute new invariants from the path integral.

A progress introduced by the present work is a rather systematic way for having topological gravities in higher dimensions. A promising case is in eight dimensions, for which [3] suggests that one can use the octonionic gravitational instanton equation [17] as a generalization of Eq.(16),

$$
\omega^{a b}-\frac{1}{2} \Omega^{a b c d} \omega^{c d}=0
$$

where $\Omega_{4}$ is the octonionic four-form used in [3].

It is indeed well known that, on an eight-dimensional manifold, one has a $\operatorname{Spin}(7)$ invariant decomposition of two-forms, as $\mathbf{2 8}=\mathbf{7} \oplus \mathbf{2 1}$, corresponding respectively to the eigenvalues $\lambda=-3,1$ of the octonionic four-form $\Omega_{4}$. Eq.(37) corresponds to setting to zero the components of the curvature in the former seven dimensional subspace. Following the same line of reasoning as in Sect.3, one foresees the following generalization of the four dimensional

\footnotetext{
${ }^{4}$ An explicit evaluation of the zero-modes of the Eguchi-Hanson gravitational instanton can be found in [15].
} 
lagrangian:

$$
\int_{\mathcal{M}_{4}} \omega^{a b^{-}} \wedge \omega^{a c^{-}} \wedge e^{b} \wedge e^{c} \rightarrow \int_{\mathcal{M}_{8}} \Omega_{4} \wedge \omega^{a b^{-}} \wedge \omega^{a c^{-}} \wedge e^{b} \wedge e^{c}
$$

This is a well-defined lagrangian on manifolds with $\operatorname{Spin}(7)$ holonomy. It is thus tempting to consider a cohomological theory, whose BRST symmetry is associated to a $\operatorname{Spin}(7) \times$ diffeomorphism $\times$ gauge invariance, corresponding to the following generalization of the four dimensional case

$$
\begin{array}{r}
\int_{\mathcal{M}_{4}} s\left(\bar{\Psi}^{a b^{-}} \wedge\left(\omega^{a c^{-}}+b^{a c^{-}}\right) \wedge e^{b} \wedge e^{c}\right) \\
\rightarrow \int_{\mathcal{M}_{8}} \Omega_{4} \wedge s\left(\bar{\Psi}^{a b^{-}} \wedge\left(\omega^{a c^{-}}+b^{a c^{-}}\right) \wedge e^{b} \wedge e^{c}\right) .
\end{array}
$$

Manifolds with $\operatorname{Spin}(7)$ and $G_{2}$ holonomy have recently attracted a renewed interest (see, e.g., [18]). The relevant rôle, which is played in this context by the self-duality conditions on the spin connection, has been underlined in [19]. We are currently studying the link between the action (39) and that of supergravity in eight dimensions. Moreover, the dimensional reduction of the theory stemming from the action (39) provides very interesting models in lower dimensions, in the spirit of [3]. For example, the reduction to seven dimensions should be related to $G_{2}$ holonomy manifolds. We can also expect an interesting low-energy effective theory for $N=2$ supergravity in four, two and zero dimensions.

Acknowledgments: We thank M. Bellon, I. Singer and R. Stora for very useful discussions.

\section{References}

[1] L. Baulieu, C. Laroche, Mod.Phys.Lett. A13 (1998) 1115, hepth/9801014.

[2] T. Eguchi, A.J. Hanson, Ann.Phys. 120 (1978) 82 .

[3] L. Baulieu, H. Kanno, I.M. Singer, Commun.Math.Phys. 194 (1998) 149, hep-th/9704167; L. Baulieu, P. West, Phys.Lett. B436 (1998) 97, hep-th/9805200. 
[4] B.S.Acharya, M. O'Loughlin, B. Spence, Nucl.Phys. B503 (1997) 657, hep-th/9705138.

[5] E. Witten, Math.Res.Lett. 1 (1994) 769, hep-th/9411102.

[6] L. Baulieu, Phys.Lett. B288 (1992) 59, hep-th/9206019.

[7] D. Anselmi, P. Frè, Nucl.Phys. B392 (1993) 401, hep-th/9208029.

[8] L. Baulieu, M.B. Green, E. Rabinovici, Nucl.Phys. B498 (1997) 119 , hep-th/9611136; Phys.Lett. B386 (1996) 91, hep-th/9606080.

[9] L. Baulieu, M. Bellon, Nucl.Phys. B266 (1986) 75.

[10] L. Baulieu, Int.J.Mod.Phys. A10 (1995) 4483, hep-th/9504015.

[11] E. Witten, Commun.Math.Phys. 117 (1988) 353.

[12] R. E. Kallosh, Nucl.Phys. B141 (1978) 141; N. K. Nielsen, Nucl.Phys. B140 (1978) 499.

[13] L. Baulieu, P. A. Grassi, D. Zwanziger, Nucl.Phys. B597 (2001) 583, hep-th/0006036.

[14] E. Witten, Phys.Lett. B206 (1988) 601.

[15] S. W. Hawking, C. N. Pope, Nucl.Phys. B146 (1978) 381; M. Bianchi, F. Fucito, G. C. Rossi, M. Martellini, Nucl.Phys. B440 (1995) 129, hepth/9409037; D. Anselmi, Nucl.Phys. B439 (1995) 617, hep-th/9411049.

[16] L. Baulieu, I. M. Singer, Nucl.Phys.Proc.Suppl. B5 (1988) 12.

[17] E.G. Floratos, A. Kehagias, Phys.Lett. B445 (1998) 69, hepth/9802107; I. Bakas, E. Floratos, A. Kehagias, Phys.Lett. B427 (1998) 283, hep-th/9810042.

[18] M. Cvetic, G. W. Gibbons, H. Lu, C. N. Pope, hep-th/0108245; H. Kanno, Y. Yasui, hep-th/0108226.

[19] A. Bilal, J. P. Derendinger, K. Sfetsos, hep-th/0111274. 